\title{
SYMPLECTIC RESOLUTIONS FOR CONICAL SYMPLECTIC VARIETIES
}

\author{
MICHEL BRION AND BAOHUA FU
}

\begin{abstract}
We introduce the notion of a conical symplectic variety, and show that any symplectic resolution of such a variety is isomorphic to the Springer resolution of a nilpotent orbit closure in a semisimple Lie algebra, composed with a linear projection.
\end{abstract}

\section{INTRODUCTION}

Definition 1. A conical symplectic variety is an affine variety $W \subset \mathbb{C}^{N}$, smooth in codimension 1 , such that (i) $W$ is invariant under the dilation action of $\mathbb{C}^{*}$ on $\mathbb{C}^{N}$, (ii) There exists a holomorphic symplectic form $\omega$ on $W_{\text {reg }}$ such that $\lambda^{*} \omega=\lambda \omega$ for all $\lambda \in \mathbb{C}^{*}$, (iii) For any resolution $\pi: Z \rightarrow W$, the 2 -form $\pi^{*} \omega$ (defined on $\pi^{-1}\left(W_{\text {reg }}\right)$ ) extends to a regular 2 -form $\Omega$ on $Z$. A resolution $\pi: Z \rightarrow W$ is called symplectic if $\Omega$ is a holomorphic symplectic form on the whole of $Z$.

For a conical symplectic variety $W \subset \mathbb{C}^{N}$, its normalization $\tilde{W}$ becomes a symplectic variety in the sense of Beauville B2]. Typical examples of conical symplectic varieties are nilpotent orbit closures $\overline{\mathcal{O}}$ in a semi-simple Lie algebra $\mathfrak{g}$ and their symplectic resolutions can be constructed as follows. For a flag variety $G / P$, its cotangent bundle $T_{G / P}^{*}$ admits a natural Hamiltonian $G$-action, and the image of the moment map $T_{G / P}^{*} \rightarrow \mathfrak{g}^{*} \simeq \mathfrak{g}$ is a nilpotent orbit closure $\overline{\mathcal{O}}_{P}$ (the Richardson orbit of $P$ ). Hence we have a projective generically finite morphism $\mu: T_{G / P}^{*} \rightarrow \overline{\mathcal{O}}_{P}$, which is called the Springer map associated to $G / P$. If $\mu$ is birational, it becomes a symplectic resolution of $\overline{\mathcal{O}}_{P}$, which we call a Springer resolution. In $[\mathrm{F}$, it is proven that symplectic resolutions of nilpotent orbit closures are exactly Springer resolutions.

Further examples of conical symplectic varieties can be constructed as follows: take a nilpotent orbit $\mathcal{O} \subset \mathfrak{g}$ and a linear subspace $L \subset \mathfrak{g}$ such that the natural projection $\mathfrak{g} \rightarrow \mathfrak{g} / L$ maps $\overline{\mathcal{O}}$ birationally to a subvariety $W$. Then $W$ is a conical symplectic variety provided it is smooth in codimension 1 . This happens for example if $L$ is a line, not contained in the secant variety of $\overline{\mathcal{O}}$. We suspect that this construction gives all conical symplectic varieties.

The main result of this note classifies symplectic resolutions for conical symplectic varieties. It generalizes the main theorem of [F], with a simpler proof.

Theorem 2. Let $\pi: Z \rightarrow W$ be a symplectic resolution for a conical symplectic variety. Then

(i) $Z \simeq T_{G / P}^{*}$ for some flag variety $G / P$.

(ii) The Springer map $\mu: T_{G / P}^{*} \rightarrow \overline{\mathcal{O}}_{P}$ associated to $G / P$ is birational.

(iii) There exists a linear subspace $L \subset \mathfrak{g}$ such that $W \subset \mathbb{C}^{N}$ is the image of $\overline{\mathcal{O}}_{P} \subset \mathfrak{g}$ under the projection $p: \mathfrak{g} \rightarrow \mathfrak{g} /$ L. The induced map $\overline{\mathcal{O}}_{P} \rightarrow W$ is birational. 
(iv) The map $\pi$ is isomorphic to the composition $p \circ \mu$.

(v) If $W$ is normal, then $W=\overline{\mathcal{O}}_{P}$.

Remark that our assumptions in Definition 1 are optimal for Theorem 2. This theorem is inspired by the alternative proof of [F] provided by Namikawa in Section 8 of [N2]. As an application, we provide a simpler proof of the main theorem of [N2], Section 7 .

Acknowledgements: We are grateful to Yoshinori Namikawa for pointing out an error in a previous version, and to the referees for their helpful comments. Baohua $\mathrm{Fu}$ is supported by NSFC (11031008 and 11225106).

\section{Proof of Theorem 2}

For a smooth variety $Y$, we denote by $T_{Y}$ the tangent bundle, by $T_{Y}^{*}$ the cotangent bundle, and by $K_{Y}$ the canonical bundle (the determinant of $T_{Y}^{*}$ ). A contact structure on $Y$ is a corank one subbundle $F \subset T_{Y}$ such that the bilinear form on $F$ with values in the quotient line bundle $L=T_{Y} / F$ deduced from the Lie bracket on $T_{Y}$ is everywhere non-degenerate. This implies that $\operatorname{dim} Y=2 n-1$ is odd and $K_{Y} \simeq L^{-n}$. We call $L$ the contact line bundle of the contact structure on $Y$. A typical example of contact manifold is the projectivised cotangent bundle $\mathbb{P}\left(T_{M}^{*}\right):=\left(T_{M}^{*} \backslash\right.$ (zero section)) $/ \mathbb{C}^{*}$ of a smooth variety $M$, where the contact line bundle is $\mathcal{O}_{\mathbb{P}\left(T_{M}^{*}\right)}(1)$.

Proposition 3. Let $X \subset \mathbb{P}^{N-1}$ be a closed singular subvariety and $f: Y \rightarrow X$ a resolution. Assume that $Y$ has a contact structure with the contact line bundle $f^{*} \mathcal{O}_{X}(1)$. Let $\tilde{X} \rightarrow X$ be the normalization map. Then $Y \simeq \mathbb{P}\left(T_{G / P}^{*}\right)$ for some flag variety $G / P$ and the induced map $Y \rightarrow \tilde{X}$ yields the Stein factorization of the projectivised Springer map associated to $G / P$.

Proof. As $X$ is singular, we get $b_{2}(Y) \geq 2$. Note that $K_{Y}=f^{*} \mathcal{O}_{X}(-n)$ with $n=$ $(\operatorname{dim} X+1) / 2$, hence $K_{Y}$ is not nef. By [KPSW], we deduce that $Y \simeq \mathbb{P}\left(T_{M}^{*}\right)$ for some smooth projective variety $M$ and $\mathcal{O}_{\mathbb{P}\left(T_{M}^{*}\right)}(1) \simeq f^{*} \mathcal{O}_{X}(1)$. This implies that $\mathcal{O}_{\mathbb{P}\left(T_{M}^{*}\right)}(1)$, hence the tangent bundle $T_{M}$ is globally generated. We deduce that $M$ is a homogeneous variety, hence by $[\mathrm{BR}]$ it is isomorphic to $G / P \times A$, where $G / P$ is a flag variety for some semisimple algebraic group $G$ and $A$ is an abelian variety.

Let $\hat{X} \subset \mathbb{C}^{N}$ be the affine cone of $X$. As $f^{*} \mathcal{O}_{X}(-1)=\mathcal{O}_{\mathbb{P}\left(T_{M}^{*}\right)}(-1)$, the map $f$ pulls back the $\mathbb{C}^{*}$-bundle $\hat{X} \backslash\{0\} \rightarrow X$ to the $\mathbb{C}^{*}$-bundle $T_{M}^{*} \backslash$ (zero section) $\rightarrow \mathbb{P}\left(T_{M}^{*}\right)$, which gives a birational map $\hat{f}: T_{M}^{*} \backslash$ (zero section) $\rightarrow \hat{X} \backslash\{0\}$. Note that $T_{M}^{*} \simeq$ $T_{G / P}^{*} \times \mathbb{C}^{g} \times A$, where $g=\operatorname{dim} A$. As $\hat{X}$ is affine, the map $\hat{f}$ contracts $\{x\} \times A$ to one point for any $x \in\left(T_{G / P}^{*} \backslash\right.$ (zero section $\left.)\right) \times\left(\mathbb{C}^{g} \backslash\{0\}\right)$, hence $\hat{f}$ factors through $T_{M}^{*} \backslash($ zero section $) \rightarrow\left(T_{G / P}^{*} \backslash\right.$ (zero section $\left.)\right) \times\left(\mathbb{C}^{g} \backslash\{0\}\right)$. As $\hat{f}$ is birational, we get $g=0$ and $M \simeq G / P$. If $\operatorname{dim} G / P=1$, then $G / P \simeq \mathbb{P}^{1}$ and $X$ is smooth, which contradicts our assumption. Thus, $\operatorname{dim} G / P \geq 2$.

Let $\mu: T_{G / P}^{*} \rightarrow \overline{\mathcal{O}}$ be the Springer map associated to $G / P$; this is a projective, generically finite morphism. By the Stein factorization, it follows that the algebra $A:=H^{0}\left(T_{G / P}^{*}, \mathcal{O}_{T_{G / P}^{*}}\right)$ is finitely generated, and $\mu$ factors as a birational morphism $\tilde{\mu}: T_{G / P}^{*} \rightarrow V:=\operatorname{Spec}(\mathrm{A})$ followed by a finite morphism $\eta: V \rightarrow \overline{\mathcal{O}}$. Note that $\tilde{\mu}$ and $\eta$ are both $G \times \mathbb{C}^{*}$-equivariant; also, $V^{\mathbb{C}^{*}}$ is a single point, say $o$. 
Let $\mathbb{C}[\hat{X}]$ be the coordinate ring of the affine variety $\hat{X}$, then the birational morphism $\hat{f}: T_{G / P}^{*} \backslash$ (zero section) $\rightarrow \hat{X} \backslash\{0\} \subset \hat{X}$ induces a homomorphism of $\mathbb{C}$ algebras $\mathbb{C}[\hat{X}] \rightarrow H^{0}\left(T_{G / P}^{*} \backslash\right.$ (zero section), $\left.\mathcal{O}\right)=A$, where the latter equality follows from $\operatorname{dim}(G / P) \geq 2$. This gives a morphism $V \rightarrow \hat{X}$ and the map $\tilde{\mu}$ (restricted to $T_{G / P}^{*} \backslash$ (zero section)) yields the Stein factorization of $\hat{f}$. Hence we get that $V \backslash\{o\}$ is the normalization of $\hat{X} \backslash\{0\}$. By taking the projectivisation, we get our claim.

We now classify conical symplectic varieties with only isolated singularities.

Lemma 4. Let $W$ be a conical symplectic variety with only isolated singularities. Then $W=\overline{\mathcal{O}}_{\text {min }}$, where $\mathcal{O}_{\text {min }}$ is the minimal nilpotent orbit in a simple Lie algebra.

Proof. As $W$ is invariant by the $\mathbb{C}^{*}$-action, it has a unique singular point, which is $\{0\}$. As $W \backslash\{0\} \rightarrow \mathbb{P} W$ is a $\mathbb{C}^{*}$-bundle, we deduce that $\mathbb{P} W$ is smooth. Note that by Lemma 1.4 [B1], the symplectic form on $W \backslash\{0\}$ induces a contact structure on $\mathbb{P} W$ with contact line bundle $\mathcal{O}_{\mathbb{P} W}(1)$. As $\mathcal{O}_{\mathbb{P} W}(1)$ is very ample, we deduce that $\mathbb{P} W \simeq \mathbb{P} \mathcal{O}_{\text {min }}$ by Cor. $1.8[\mathrm{~B} 1]$. This gives that $W=\overline{\mathcal{O}}_{\text {min }}$.

Now let us prove Theorem 2. First note that the $\mathbb{C}^{*}$-action on $W$ lifts to $Z$ (see for example Prop. A.7 of [N1]), which makes $\pi$ to be $\mathbb{C}^{*}$-equivariant. Let $\Omega$ be the symplectic form on $Z$ extending $\pi^{*} \omega$, then we have $\lambda^{*} \Omega=\lambda \Omega$. We denote by $\mathbb{P} Z$ the quotient $\left(Z \backslash \pi^{-1}(0)\right) / \mathbb{C}^{*}$. Then we get a morphism $\bar{\pi}: \mathbb{P} Z \rightarrow \mathbb{P} W$. By Sections 3 and 4 of [N2], we have

Lemma 5. $\mathbb{P} Z$ is a smooth contact projective variety with the contact line bundle $L:=\bar{\pi}^{*} \mathcal{O}_{\mathbb{P} W}(1)$.

If $\mathbb{P} W$ is smooth, then by Lemma 4, $W=\overline{\mathcal{O}}_{\text {min }} \subset \mathfrak{g}$. As $W$ admits a symplectic resolution, this implies that $\mathfrak{g}$ is of type $A$ and $Z \simeq T_{\mathbb{P}^{n}}^{*}$ (cf. [F] or Proposition [6] below). Assume now that $\mathbb{P} W$ is singular, then we can apply Lemma 5 and Proposition 3 to conclude that $(\mathbb{P} Z, L) \simeq\left(\mathbb{P}\left(T_{G / P}^{*}\right), \mathcal{O}_{\mathbb{P}\left(T_{G / P}^{*}\right)}(1)\right)$. Note that we may take $G=\operatorname{Aut}^{0}(G / P)$, up to changing $G$ and $P$. By the proof of Proposition 3 , we have the following diagram:

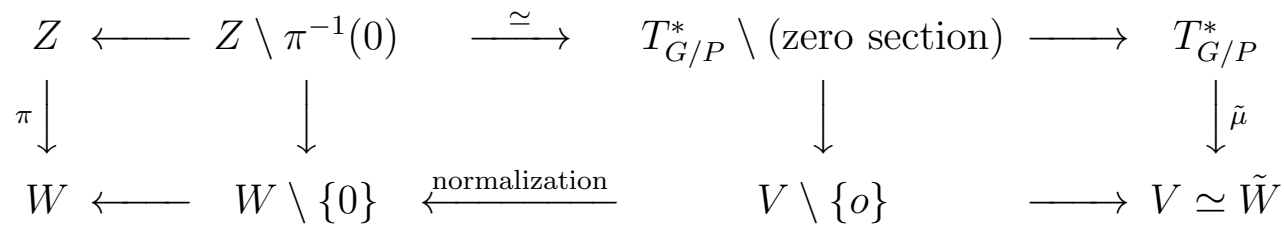

As $\mathbb{P} W$ is not smooth, we may assume $G / P \neq \mathbb{P}^{n}$. Now we can apply Lemma 5 [N2] and the argument in loc. cit. (p. 183) to deduce that $Z \simeq T_{G / P}^{*}$ and this identifies $\tilde{\pi}: Z \rightarrow \tilde{W}$ with the map $\tilde{\mu}$ from the Stein factorization of the Springer map $\mu: T_{G / P}^{*} \rightarrow \overline{\mathcal{O}}$. As seen in the proof of Proposition 3 , it follows that $\tilde{W}$ admits a $G \times \mathbb{C}^{*}$-action such that $\tilde{\pi}$ is equivariant.

As $W \subset \mathbb{C}^{N}$ and $\mathbb{C}^{*}$ acts on $\mathbb{C}^{N}$ by dilations, the coordinate ring $\mathbb{C}[W]$ is a subalgebra of $\mathbb{C}[\tilde{W}]$ generated by elements of degree 1 . On the other hand, we have $\mathbb{C}[\tilde{W}]=H^{0}\left(T_{G / P}^{*}, \mathcal{O}_{T_{G / P}^{*}}\right)$. The space of degree 1 elements in $H^{0}\left(T_{G / P}^{*}, \mathcal{O}_{T_{G / P}^{*}}\right)$ is $H^{0}\left(G / P, T_{G / P}\right)$, which is $\mathfrak{a} \mathfrak{u t}(G / P)=\mathfrak{g}$. Thus, the algebra $\mathbb{C}[W]$ is generated by a linear subspace of $\mathfrak{g}$, that we view as the orthogonal of a linear subspace $L \subset \mathfrak{g}$. So 
the normalization map $\tilde{W} \rightarrow W$ factors as the map $\tilde{W} \rightarrow \overline{\mathcal{O}} \subset \mathfrak{g} \cong \mathfrak{g}^{*}$ corresponding to the inclusion $\mathfrak{g} \subset H^{0}\left(T_{G / P}^{*}, \mathcal{O}_{T_{G / P}^{*}}\right)$, followed by a map $p: \overline{\mathcal{O}} \rightarrow W$, the restriction of the quotient map $\mathfrak{g} \rightarrow \mathfrak{g} / L$. Thus $p$ is birational. If $W$ is normal, then $W=\tilde{W}$, hence we get $W=\overline{\mathcal{O}}$. This finishes the proof of Theorem 2 .

In the proof of Theorem 2, we used $[\mathrm{F}]$ to deduce that among minimal nilpotent orbit closures, only that of type $A$ admits a symplectic resolution. One can in fact prove a stronger result.

Proposition 6. Let $W$ be a normal variety of dimension $2 n \geq 4$ with an isolated singularity 0 and a contracting $\mathbb{C}^{*}$-action. Assume that $W \backslash\{0\}$ admits a symplectic form $\omega$ satisfying $\lambda^{*} \omega=\lambda \omega, \forall \lambda \in \mathbb{C}^{*}$. If $W$ admits a symplectic resolution $\pi: Z \rightarrow$ $W$, then $Z \simeq T_{\mathbb{P}^{n}}^{*}$ and $W \simeq \overline{\mathcal{O}}_{\min } \subset \mathfrak{s l}_{n+1}$.

Proof. Recall that the action of $\mathbb{C}^{*}$ on $W$ lifts to an action on $Z$ (see Prop. A.7 of [N1]). Let $Z^{\mathbb{C}^{*}}$ be the subvariety of $Z$ consisting of $\mathbb{C}^{*}$-fixed points, which is a disjoint union of smooth subvarieties since $Z$ is smooth. As $W^{\mathbb{C}^{*}}=\{0\}$, we have that $Z^{\mathbb{C}^{*}}$ is contained in the fiber $\pi^{-1}(0)$, hence it is a union of projective manifolds.

By the Bialynicki-Birula decomposition (see [BB] which applies in this non-projective setting, since $Z$ is proper over $W$ which is contracted to 0 by the $\mathbb{C}^{*}$-action), there exists an irreducible component $M$ of $Z^{\mathbb{C}^{*}}$ such that the set $U:=\left\{z \in Z \mid \lim _{\lambda \rightarrow 0} \lambda \cdot z \in\right.$ $M\}$ is open in $Z$. Let $\Omega$ be the symplectic form on $Z$, which is the extension of $\pi^{*} \omega$. As $\lambda^{*} \Omega=\lambda \Omega$ for all $\lambda \in \mathbb{C}^{*}$, we obtain that $(U, \Omega) \simeq\left(T_{M}^{*}, \omega_{\text {can }}\right)$ as $\mathbb{C}^{*}$-varieties (cf. Lemma $3.7[\mathrm{~F}]$ ), where $\omega_{\text {can }}$ is the canonical symplectic form on $T_{M}^{*}$. On the other hand, we have $\operatorname{dim} \pi^{-1}(0) \leq \frac{1}{2} \operatorname{dim} W=\operatorname{dim} M$ (Cor. 8.5 [CMSB]). This implies that $M$ is an irreducible component of $\pi^{-1}(0)$. As $M$ is smooth, by Cor. 8.7 [CMSB], we get that $M \simeq \mathbb{P}^{n}$. It follows (for example by the arguments above) that the map $U \rightarrow W$ is the Springer map $\mu: T_{\mathbb{P}^{n}}^{*} \rightarrow \overline{\mathcal{O}}_{\text {min }} \subset \mathfrak{s l}_{n+1}$. As $\mu$ is projective, we get $U=Z$.

\section{An APPLICATION}

A symplectic variety is a normal variety $W$ with a symplectic form $\omega$ on its smooth locus such that for any resolution $\phi: Z \rightarrow W$, the 2 -form $\phi^{*} \omega$ defined on $\phi^{-1}\left(W_{\text {reg }}\right)$ extends to a regular 2 -form on $Z$.

As an application of Theorem 2, we provide an alternative proof of the following main result of [N2].

Theorem 7 (Namikawa). Let $(W, \omega)$ be a singular symplectic variety embedded in $\mathbb{C}^{N}$ as a complete intersection of hypersurfaces defined by homogeneous polynomials. Assume that the symplectic form $\omega$ satisfies $\lambda^{*} \omega=\lambda^{k} \omega, \forall \lambda \in \mathbb{C}^{*}$ for some $k$. Then $(W, \omega)$ is isomorphic to the nilpotent cone $\left(\mathcal{N}, \omega_{K K}\right)$ of a semisimple complex Lie algebra $\mathfrak{g}$ together with the Kirillov-Kostant form $\omega_{K K}$.

Proof. By Section 2 of [N2], $W$ is a conical symplectic variety with a symplectic resolution, hence by Theorem 2 , we get that $(W, \omega)$ is a nilpotent orbit closure in a semi-simple Lie algebra $\mathfrak{g}$. But every nilpotent orbit closure $\overline{\mathcal{O}} \subset \mathfrak{g}$ which is a complete intersection in $\mathfrak{g}$ must be the full nilpotent cone, by the main result of 
Section 7 of [N2]. We now provide a proof of that result, which is somehow shorter and more uniform than the original one.

Let $d_{1}, \cdots, d_{r} \geq 2$ be the degrees of defining equations of $\overline{\mathcal{O}}$ in $\mathfrak{g}$. Then $r=$ $\operatorname{codim}_{\mathfrak{g}}(\mathcal{O})$ and by [N2] (Section 2, p. 160), we have

$$
\sum_{i=1}^{r} d_{i}=\frac{1}{2} \operatorname{dim} \mathcal{O}+\operatorname{codim}_{\mathfrak{g}}(\mathcal{O}), \quad \text { and } \quad \operatorname{codim}_{\mathfrak{g}}(\mathcal{O}) \leq \frac{1}{3} \operatorname{dim} \mathfrak{g} .
$$

We may assume that $\mathfrak{g}$ is simple. We denote by $I$ the ideal of $\overline{\mathcal{O}}$ in the coordinate ring $\mathbb{C}[\mathfrak{g}]$, and by $\mathfrak{m}$ the maximal ideal of 0 in $\mathbb{C}[\mathfrak{g}]$. Let $G$ be the adjoint group of $\mathfrak{g}$; then $G \times \mathbb{C}^{*}$ acts on $\mathfrak{g}$ (where $\mathbb{C}^{*}$ acts by dilations) and stabilizes $\overline{\mathcal{O}}$ and 0 . Hence $G \times \mathbb{C}^{*}$ acts on $\mathbb{C}[\mathfrak{g}]$ and stabilizes $I \subset \mathfrak{m}$. Since $G \times \mathbb{C}^{*}$ is reductive, we may find a submodule $M \subset I$ which is mapped isomorphically to $I / \mathfrak{m} I$ under the quotient map $I \rightarrow I / \mathfrak{m} I$. By the graded Nakayama lemma, a homogeneous basis of $M$ yields a minimal generating system of the ideal $I$, and hence a regular sequence in $\mathbb{C}[\mathfrak{g}]$ since $\overline{\mathcal{O}}$ is a complete intersection. In geometric terms, the morphism

$$
f: \mathfrak{g} \longrightarrow M^{*}=: V
$$

corresponding to the inclusion $M \subset \mathbb{C}[\mathfrak{g}]$ is flat and its (scheme-theoretic) fiber $f^{-1}(0)$ equals $\overline{\mathcal{O}}$ (this is a slightly more precise version of Lemma 3 in [N2]). Thus, $f$ is open, and hence surjective by $\mathbb{C}^{*}$-equivariance.

Choose a maximal torus $T \subset G$ and denote by $\mathfrak{t}$ its Lie algebra; this is a Cartan subalgebra of $\mathfrak{g}$. Since $G \mathfrak{t}$ is dense in $\mathfrak{g}$, there exists $x \in \mathfrak{t}$ such that the differential $d f_{x}: \mathfrak{g} \rightarrow V$ is surjective. As $d f_{x}$ is linear and $T$-equivariant, it follows that each weight of the $T$-module $V$ is also a weight of $\mathfrak{g}$. In particular, the highest weight of any simple summand of the $G$-module $V$ is either the highest root, or the highest short root $\lambda$ (if $G$ is not simply laced), or 0 . Moreover, the highest root cannot occur, since the corresponding simple module is just $\mathfrak{g}$, and $\operatorname{dim}(V)=\operatorname{dim}(\mathfrak{g})-\operatorname{dim}(\overline{\mathcal{O}})<\operatorname{dim}(\mathfrak{g})$.

If $G$ is simply laced, then $V$ must be the trivial $G$-module, and hence $\overline{\mathcal{O}}$ contains the nilpotent cone $\mathcal{N}$; so we conclude that $\overline{\mathcal{O}}=\mathcal{N}$. Thus, we may assume that $G$ is not simply laced; then we have an isomorphism of $G$-modules

$$
V \cong p V(0) \oplus q V(\lambda)
$$

where $p, q$ are non-negative integers, $V(0)$ denotes the trivial $G$-module $\mathbb{C}$, and $V(\lambda)$, the simple $G$-module with highest weight $\lambda$. (The latter module is called the "little adjoint module" in $[\mathrm{P}$, where its invariant theoretical properties are investigated.) If $q=0$ then we conclude as above that $\overline{\mathcal{O}}=\mathcal{N}$; thus, we may further assume that $q \geq 1$.

If $G$ of type $C_{n}$ (resp. $F_{4}, G_{2}$ ), then $V(\lambda)$ has dimension $2 n^{2}-n-1$ (resp. 26, 7), which contradicts the inequality $\operatorname{codim}_{\mathfrak{g}}(\mathcal{O})=\operatorname{dim} V \leq \frac{1}{3} \operatorname{dim}(\mathfrak{g})$ in (3.1). Thus, we may assume that $G=\operatorname{SO}(2 n+1)$; then $V(\lambda)$ is the natural $G$-module $\mathbb{C}^{2 n+1}$.

Since $f$ is surjective and $G \mathfrak{g}^{T}$ is dense in $\mathfrak{g}$, it follows that $G V^{T}$ is dense in $V$. But this does not hold for $V=2 V(\lambda)$ (as $V(\lambda)^{T}$ is a line), and hence $q \leq 1$. So we may take $V=p V(0) \oplus V(\lambda)$. Since the algebra of invariant functions $\mathbb{C}[V(\lambda)]^{G}$ is generated by the quadratic form defining $G$, it follows that the categorical quotient $V / / G:=\operatorname{Spec} \mathbb{C}[\mathrm{V}]^{\mathrm{G}}$ is an affine space, and the quotient morphism

$$
q_{V}: V \longrightarrow V / / G
$$


is flat with reduced fibers. Since $q_{V}$ sits in a commutative square

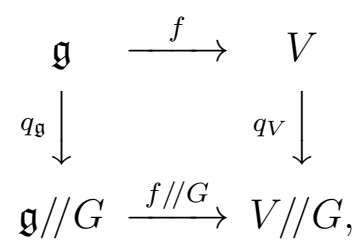

where $f, q_{\mathfrak{g}}$ are flat with reduced fibers, we see that $f / / G$ is flat with reduced fibers, too.

We claim that $f / / G$ is also finite. Consider indeed the restriction $f^{T}: \mathfrak{g}^{T}=$ $\mathfrak{t} \rightarrow V^{T}$. Then the fiber of $f^{T}$ at 0 equals $\overline{\mathcal{O}} \cap \mathfrak{t}=\{0\}$ (as a set). Since $f^{T}$ is equivariant for the natural actions of $\mathbb{C}^{*}$, it follows that $f^{T}$ is finite. Hence so is $f^{T} / W: \mathfrak{g}^{T} / W \rightarrow V^{T} / W$, where $W$ denotes the Weyl group of $(G, T)$. But the natural map $\mathfrak{g}^{T} / W \rightarrow \mathfrak{g} / / G$ is an isomorphism by the Chevalley restriction theorem; moreover, the analogous map $V^{T} / W \rightarrow V / / G$ is finite, and dominant since $G V^{T}$ is dense in $V$. The finiteness of $f / / G$ follows from this in view of the commutative square

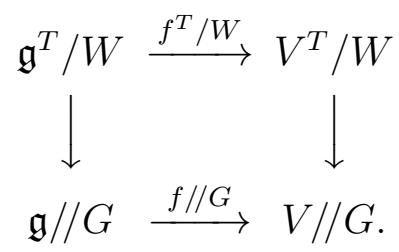

Since $f / / G$ is also flat with reduced fibers, it is a finite étale cover, and hence an isomorphism as $V / / G$ is an affine space. In other words, the algebra $\mathbb{C}[\mathfrak{g}]^{G}$ is freely generated by the pull-backs under $f$ of the $p$ projections $V \rightarrow V(0)$ and of the basic invariant of $V(\lambda)$. The degrees of these invariants are $a_{1}, \ldots, a_{p}, 2 a_{p+1}$, where $a_{1}, \ldots, a_{p}$ denote the weights of the action of $\mathbb{C}^{*}$ on $p V(0)$, and $a_{p+1}$ the weight of that action on $V(\lambda)$. On the other hand, the degrees of the basic invariants of $\mathbb{C}[\mathfrak{g}]^{G}$ are $2,4, \ldots, 2 n$. In particular, we have $n=p+1$ and

$$
a_{1}+\cdots+a_{n-1}+2 a_{n}=n^{2}+n .
$$

This implies that $r=\operatorname{dim} V=3 n$ and $\operatorname{dim} \mathcal{O}=\operatorname{dim} \mathfrak{g}-3 n=2 n^{2}-2 n$. Moreover, we may assume that the degrees of defining equations of $\overline{\mathcal{O}}$ satisfy $d_{i}=a_{i}$ for $i=$ $1, \cdots, n-1$, and $d_{n}=\cdots=d_{3 n}=a_{n}$. By (3.1), we have $d_{1}+\cdots+d_{3 n}=\left(n^{2}-n\right)+3 n$, which gives that

$$
a_{1}+\cdots+a_{n-1}+(2 n+1) a_{n}=n^{2}+2 n .
$$

Combining the two displayed equalities yields $(2 n-1) a_{n}=n$ which is not possible since $a_{n}=d_{n} \geq 2$.

\section{REFERENCES}

[BB] Bialynicki-Birula, A.: Some theorems on actions of algebraic groups, Ann. of Math., II. Ser. 98 (1973), 480-497

[B1] Beauville, A.: Fano contact manifolds and nilpotent orbits, Comment. Math. Helv. 73 (1998), no. 4, 566-583

[B2] Beauville, A.: Symplectic singularities, Invent. Math. 139 (2000), 541-549

[BR] Borel, A. and Remmert, R.: Über kompakte homogene Kählersche Mannigfaltigkeiten, Math. Ann. 145 (1961/1962), 429-439 
[CMSB] Cho, K., Miyaoka, Y. and Shepherd-Barron, N. I.: Characterizations of projective space and applications to complex symplectic manifolds, Higher dimensional birational geometry (Kyoto, 1997), 1-88, Adv. Stud. Pure Math., 35, Math. Soc. Japan, Tokyo, 2002

[F] Fu, B.: Symplectic resolutions for nilpotent orbits, Invent. Math. 151 (2003), 167-186

[KPSW] Kebekus, S., Peternell, T., Sommese, A., Wisniewski, J.: Projective contact manifolds, Invent. Math. 142 (2000), 1-15.

[N1] Namikawa, Y.: Flops and Poisson deformations of symplectic varieties, Publ. Res. Inst. Math. Sci. 44 (2008), 259-314

[N2] Namikawa,Y.: On the structure of homogeneous symplectic varieties of complete intersection, Invent. Math. 193 (2013), no. 1, 159-185

[P] Panyushev, D.: Invariant theory of the little adjoint module, J. Lie Theory 22 (2012), 803-816.

Michel Brion

Institut Fourier, Université de Grenoble, France

e-mail: Michel.Brion@ujf-grenoble.fr

Baohua $\mathrm{Fu}$

Institute of Mathematics, AMSS, 55 ZhongGuanCun East Road, Beijing, 100190, China

e-mail: bhfu@math.ac.cn 